\title{
Treatment Outcomes of External Beam Radiation Therapy for Unresectable Locally Advanced Thyroid Cancer with or without Metastasis: A Retrospective Single-Center Study
}

\author{
Yuki Wada *(D), Akira Anbai, Satoshi Kumagai, Eriko Okuyama, Noriko Takagi and Manabu Hashimoto
}

check for updates

Citation: Wada, Y.; Anbai, A.; Kumagai, S.; Okuyama, E.; Takagi, N.; Hashimoto, M. Treatment Outcomes of External Beam Radiation Therapy for Unresectable Locally Advanced Thyroid Cancer with or without Metastasis: A Retrospective Single-Center Study. Radiation 2021, 1, 174-182. https://doi.org/10.3390/ radiation1030016

Academic Editor: Jason Parsons

Received: 24 May 2021

Accepted: 6 July 2021

Published: 7 July 2021

Publisher's Note: MDPI stays neutral with regard to jurisdictional claims in published maps and institutional affiliations.

Copyright: (c) 2021 by the authors. Licensee MDPI, Basel, Switzerland. This article is an open access article distributed under the terms and conditions of the Creative Commons Attribution (CC BY) license (https:/ / creativecommons.org/licenses/by/ $4.0 /)$.
Department of Radiology, Akita University Graduate School of Medicine, 1-1-1 Hondo, Akita 010-8543, Japan; anbai@doc.med.akita-u.ac.jp (A.A.); skuma@med.akita-u.ac.jp (S.K.); eri.lily@med.akita-u.ac.jp (E.O.); tnoriko@med.akita-u.ac.jp (N.T.); hashimms@med.akita-u.ac.jp (M.H.)

* Correspondence: ywada@med.akita-u.ac.jp; Tel.: +81-18-834-1111; Fax: +81-18-836-2623

Simple Summary: Treatment effect and safety of external beam radiation therapy (EBRT) for unresectable locally advanced thyroid cancer are not established. Tyrosine kinase inhibitors (TKIs) are used in first-line treatment for unresectable thyroid cancer; however, these treatments are sometimes unsuitable for patients with poor performance status or tumors invasive to trachea or large vessels. EBRT might have potential to treat these conditions. This single-center retrospective study aimed to evaluate the treatment effect and safety of EBRT for advanced thyroid cancer. Our findings indicate that EBRT for unresectable thyroid cancer has the potential to provide a highly objective response rate without severe toxicities. Although TKIs are the choice for first-line treatment for unresectable thyroid cancer, EBRT may also be considered if TKIs are unsuitable. However, because the present study evaluated a small number of patients with heterogenous histological tumor types, our findings are not conclusive.

Abstract: We evaluated treatment outcomes of external beam radiation therapy (EBRT) for unresectable locally advanced thyroid cancer (LATC) with or without metastasis. We enrolled 11 LATC patients who underwent EBRT (median age: 76 (45-83) years; six males and five females). Eastern Cooperative Oncology Group performance statuses of $0(n=3), 1(n=1), 2(n=6)$, and $3(n=1)$ were observed. Histologic types included papillary carcinoma $(n=5)$, anaplastic carcinoma $(n=3)$, and squamous cell carcinoma $(n=3)$. The organs invaded by the tumor that caused it to be deemed unresectable were common carotid artery $(n=5)$, trachea $(n=4)$, aorta $(n=1)$ and larynx $(n=1)$. The median follow-up time was 6 months. One, seven, two, and one patient showed complete response $(\mathrm{CR})$, partial response (PR), stable disease, and progressive disease, respectively. The rate of local $\mathrm{CR}+\mathrm{PR}$ was $73 \%$; moreover, $75 \%$ of patients achieved a $>30 \%$ tumor size reduction within 6 months. The median local progression-free survival of patients with local CR+PR was 11.5 (4-68) months. The median overall survival was 6 (1-68) months. Grade 3 acute complications occurred in five (45\%) patients. No patients had Grade 4 or 5 complications. In conclusion, EBRT reduced the tumor volume in $75 \%$ of LATC patients without inducing severe toxicity. This therapy should be considered as a treatment option for LATC.

Keywords: external beam radiation therapy; locally advanced thyroid cancer; tyrosine kinase inhibitor; squamous cell thyroid cancer

\section{Introduction}

Surgical resection with or without radioactive iodine is a standard therapy for operable advanced thyroid cancer [1-3]. Unresectable thyroid cancers can be treated using three tyrosine kinase inhibitors (TKIs), namely lenvatinib, sorafenib, and vandetanib. These TKIs have shown high response rates and survival benefits in phase II/III studies [4-6]. However, locally advanced thyroid cancer (LATC) involving invasion of the trachea or neck 
vessels has a high risk for TKI-related toxicities, including trachea or vessel perforation, which renders TKIs unsuitable for use [7,8].

The role of external beam radiation therapy (EBRT) for unresectable thyroid cancer remains unclear. Studies with small patient numbers have reported benefits of EBRT for LATC [9-13]; however, most of the previous studies on EBRT assessed it as an adjuvant or salvage therapy for pathological or gross remnants of thyroid cancer after surgery. There have been no large-scale or prospective studies on EBRT for unresectable LATC. To our knowledge, only a few studies reported on EBRT for unresectable LATC, and one of them reported that locoregional disease control at 1 year was $33 \%$ in patients with gross unresectable thyroid cancer treated using EBRT with a 60-70 Gy dose [10]. Furthermore, the efficacy and safety of TKI and EBRT combination therapy, either concurrently or sequentially, remain unclear. Therefore, there is a need to evaluate the effectiveness and safety of EBRT for unresectable LATC. This retrospective study aimed to evaluate the efficacy and tolerability of EBRT for unresectable LATC.

\section{Materials and Methods}

\subsection{Ethical Statement}

This retrospective study protocol was approved by the Akita University Hospital institutional review board (no. 1951, approved on 27 April 2018). All methods were performed following the guidelines and regulations of the ethics board. The requirement for informed consent was waived since this study used anonymized data.

\subsection{Patients}

We included 11 consecutive patients with LATC who underwent EBRT between January 2002 and December 2017 in our institution. We retrospectively obtained data regarding medical history, imaging, and radiation plans. The clinical T stage was determined according to the 8th edition of the Union for International Cancer Control TNM Classification of Malignant Tumors [14].

\subsection{Evaluation}

Tumor response to therapy was evaluated according to the Response Evaluation Criteria in Solid Tumors version 1.1; furthermore, adverse events were scored according to the common terminology criteria for adverse events version 5.0. Local response, overall response, and overall survival (OS) were analyzed in all patients. Local progression-free survival (L-PFS) was examined in patients with complete response (CR) or partial response (PR) to irradiation. OS and L-PFS were defined as the time from the initiation of EBRT to death from any cause and local tumor relapse/death from any cause, respectively. The Kaplan-Meier method was used to analyze L-PFS and OS; furthermore, a waterfall plot was constructed to analyze the local response. The Mann-Whitney U test was used to analyze the effects of the irradiation dose with concurrent systemic therapy on the maximum percent change in the local tumor size. To compare irradiated dose schedules, we used $\mathrm{BED}_{10}$, which is calculated as "total dose $\times(1+$ dose per fraction $/ 10)$ " for analysis [15]. Statistical significance was set at a $p$-value $<0.05$. All analyses were performed using BellCurve for Excel (version 3.20; Tokyo, Japan).

\subsection{Radiation Therapy}

All EBRT plans were based on three-dimensional conformal radiotherapy with a computed tomography (CT) simulation scan. The target volumes were contoured on CT simulation images according to pretreatment diagnostic images, including contrastenhanced CT and ultrasound. All radiation targets included the primary thyroid tumor mass. In contrast, the inclusion of involved neck lymph nodes as a radiation target was determined by the radiation oncologist-in-charge based on the treatment intent, patient condition, and tumor condition. The gross tumor volume (GTV) was determined as the primary tumor mass with or without the involved neck lymph nodes. The clinical 
target volume (CTV) was defined as the GTV plus an approximate $0.5-1 \mathrm{~cm}$ margin in all directions or inclusion of the total thyroid body. The planning target volume was determined as the CTV plus $0.5 \mathrm{~cm}$ in all directions. The dose reference point was defined at the iso-center. The radiation dose was 50-60 Gy and was often divided into 1.8-2.5 Gy per fraction and administered over 5-6 weeks. However, a shorter radiation schedule with higher doses per fraction was used for patients depending on the decision of the radiation oncologist-in-charge. Radiation was delivered with 1-4 fields from multiple directions, with the delivery method being determined by the radiation oncologist in charge. A 4- or 6-megavoltage $\mathrm{X}$-ray beam was used for all patients. For organs at risk surrounding the thyroid, the following dose constraints were to be met as much as possible in the present study: the maximum dose of spinal cord, larynx, and esophagus were $44 \mathrm{~Gy}$, 66 Gy and $60 \mathrm{~Gy}$, respectively. Furthermore, we planned to avoid high-dose distribution area that exceeded $110 \%$ of the prescribed dose where possible.

\section{Results}

\subsection{Patient Characteristics}

Table 1 summarizes the patient characteristics. We included 11 patients (median age: 76 (45-83) years; six males and five females). Three, one, six, and one patient had an Eastern Cooperative Oncology Group performance status (PS) of 0, 1, 2, and 3, respectively. Seven $(64 \%)$ patients were in bad condition, as indicated by a PS of 2 or 3 . Regarding the histological tumor types, five, three, and three patients had papillary cell carcinomas, undifferentiated cell carcinomas, and squamous cell carcinomas, respectively. The organs invaded by the tumor that caused it to be deemed unresectable were common carotid artery $(n=5)$, trachea $(n=4)$, aorta $(n=1)$ and larynx $(n=1)$. The total radiation doses (30-60 Gy) were administered in 15-30 fractions with 1.8-2.5 Gy/fraction. The radiation targets included primary tumors with or without the involvement of neck lymph nodes. The biological effective dose at an alpha/beta of $10\left(\mathrm{BED}_{10}\right)$ of the administered doses was 36-72 Gy10 (median: $63.7 \mathrm{~Gy}_{10}$ ). Two patients prematurely stopped EBRT owing to tumor progression or worsening of patient condition. Five patients received irradiation with concurrent chemotherapy or TKI, including docetaxel $(n=2)$, cisplatin/fluorouracil/docetaxel $(n=1)$, cisplatin/etoposide $(n=1)$, and lenvatinib $(n=1)$. Four patients underwent chemotherapy or TKI (TS-1 $(n=2)$, nedaplatin/etoposide $(n=1)$, and sorafenib followed by lenvatinib $(n=1))$ after completing EBRT as adjuvant or second-line treatment for progression.

Table 1. Patient and tumor characteristics $(n=11)$.

\begin{tabular}{cc}
\hline Characteristics & Value \\
\hline Age (years) & 76 \\
Median & $45-83$ \\
Range & \\
Gender & 6 \\
Male & 5 \\
Female & \\
Performance status & 3 \\
0 & 1 \\
1 & 6 \\
2 & 1 \\
3 & 5 \\
Histology & 3 \\
Papillary carcinoma & 3 \\
Anaplastic carcinoma & \\
Squamous cell carcinoma &
\end{tabular}


Table 1. Cont.

\begin{tabular}{cc}
\hline Characteristics & Value \\
\hline Common carotid artery (T4b) & 5 \\
Aorta (T4b) & 1 \\
Trachea (T4a) & 4 \\
Larynx (T4a) & 1 \\
Tracheostomy & \\
Yes & 4 \\
No & 7 \\
Irradiation dose & \\
50 Gy in 30 fractions & 7 \\
54 Gy in 30 fractions & 1 \\
50 in 20 fractions & 1 \\
30 Gy in 23 fractions (canceled halfway) 15 fractions (canceled halfway) & 1 \\
Concurrent systemic therapy & 1 \\
Docetaxel & \\
Cisplatin/fluorouracil/docetaxel & 2 \\
Cisplatin/etoposide & 1 \\
Lenvatinib & 1 \\
\hline
\end{tabular}

Clinical T stage was according to 8th edition of the Union for International Cancer Control TNM Classification of Malignant Tumors.

\subsection{Treatment Outcomes}

The median follow-up time was 6 (range 1-68) months. The maximum percentage change in the primary tumor size is shown as a waterfall plot in Figure 1. Local treatment responses included local CR $(n=1)$, local PR $(n=7)$, stable disease (SD; $n=2)$, and progressive disease (PD; $n=1$ ). The rate of local $\mathrm{CR}+\mathrm{PR}$ was $73 \%$; among them, $75 \%$ achieved a $\geq 30 \%$ tumor size reduction within 6 months. The local CR group $(n=1)$ consisting of squamous cell carcinoma which invaded to the common carotid artery with PS 2, was treated with EBRT and TKI concurrently. The local PR group $(n=7)$ consisting of four papillary carcinoma which invaded to two each of the common carotid artery and the trachea (two each of PS 0 and PS 2) were treated with EBRT alone. Two squamous cell carcinoma which invaded to the common carotid artery or the trachea (one each of PS 0 and PS 2) were treated with concurrent use of EBRT and systemic chemotherapy. One anaplastic carcinoma which invaded to the larynx (PS 1) was treated with concurrent use of EBRT and systemic chemotherapy. The local SD group $(n=2)$ consisting of one anaplastic carcinoma which invaded to the common carotid artery (PS 3) and one papillary carcinoma which invaded to the aorta (PS2), were treated with EBRT alone. The local PD group $(n=1)$ consisting of anaplastic carcinoma which invaded to the trachea (PS 2), was treated with concurrent use of EBRT and systemic chemotherapy.

The overall treatment responses included CR $(n=1), \operatorname{PR}(n=6), \operatorname{SD}(n=1)$, and PD $(n=3)$. Two patients who achieved local PR or SD were diagnosed with lung metastases during or soon after EBRT; therefore, they were defined as PD in the overall response. One patient treated with EBRT with a concurrent lenvatinib prescription achieved local and overall CR. However, the efficacy and safety of concurrent EBRT and lenvatinib remain unclear. The patient showed uncontrollable bleeding from the thyroid tumor with skin invasion even after lenvatinib administration; therefore, EBRT for the primary tumor was initiated after the patient received detailed explanations of the expected treatment merit and complications. Regarding the maximum percentage change in the primary tumor size, there were no significant differences according to the radiation dose $\left(\mathrm{BED}_{10}<63.7 \mathrm{vs}\right.$. $\geq 63.7 \mathrm{~Gy}_{10}, p=0.18$ ) or concurrent systemic therapy (presence vs. absence, $p=0.54$ ). 


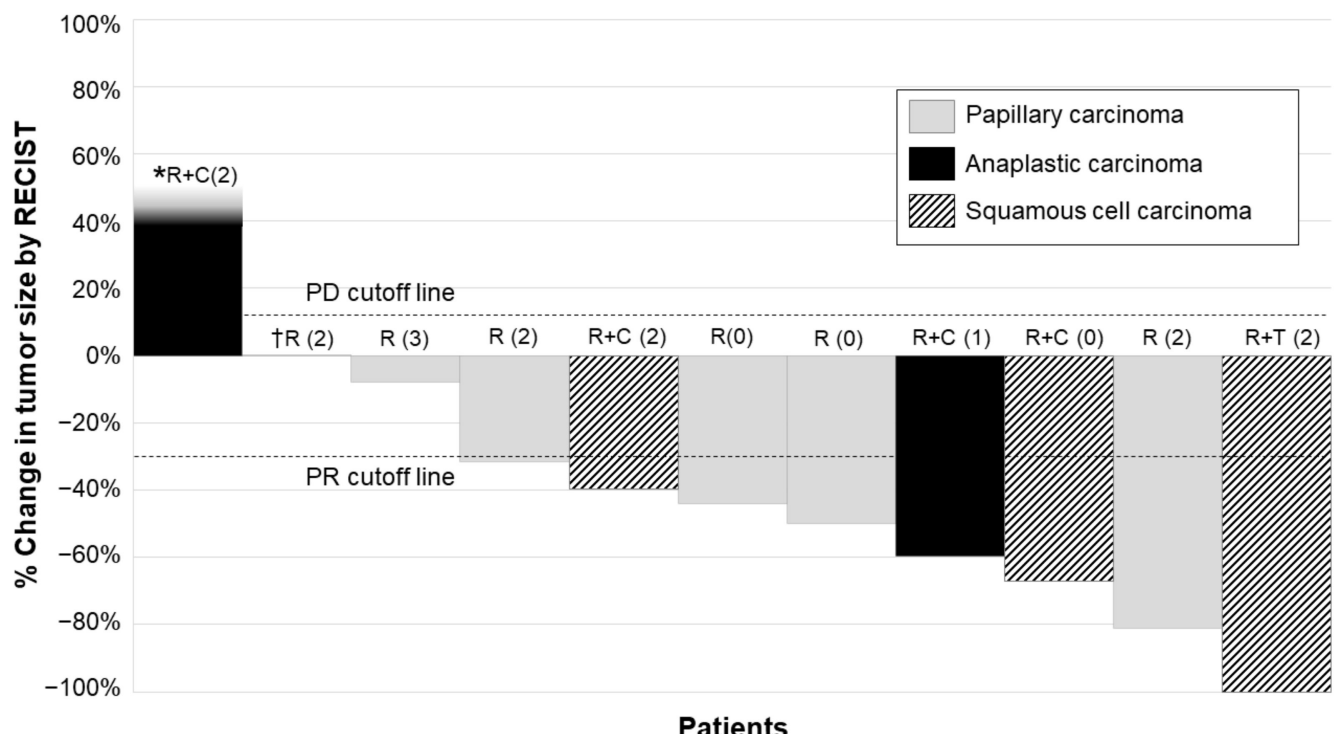

Figure 1. Waterfall plot of primary tumor response. Percentage changes in primary tumor size before and after irradiation were evaluated based on the Response Evaluation Criteria in Solid Tumors (RECIST) version 1.1. One patient ${ }^{*}$ ) showed progressive disease (PD) during EBRT with a tumor increase of $>20 \%$. One patient $(\dagger)$ diagnosed with papillary carcinoma showed a tumor size reduction of only $1 \%$. The PD and partial response (PR) cutoff lines represent $+20 \%$ and $-30 \%$, respectively, based on the RECIST. R: Treated by EBRT without concurrent use of systemic therapy. R+C: Treated by concurrent use of EBRT and systemic chemotherapy. R+T: Treated by concurrent use of EBRT and tyrosine kinase inhibitor. The number in parentheses after the " $R$ ", " $R+C$ " or " $R+T$ " indicates a performance status.

Figure 2 shows the Kaplan-Meier curves of OS in all patients and L-PFS in patients with local CR or PR with radiation. The median OS was 6 (range: 1-68) months. The median L-PFS of patients with local CR/PR was 11.5 (range: 4-68) months. One patient with clinical stage IVA papillary carcinoma who presented an overall PR survived until 68 months after EBRT without requiring any adjuvant therapy.

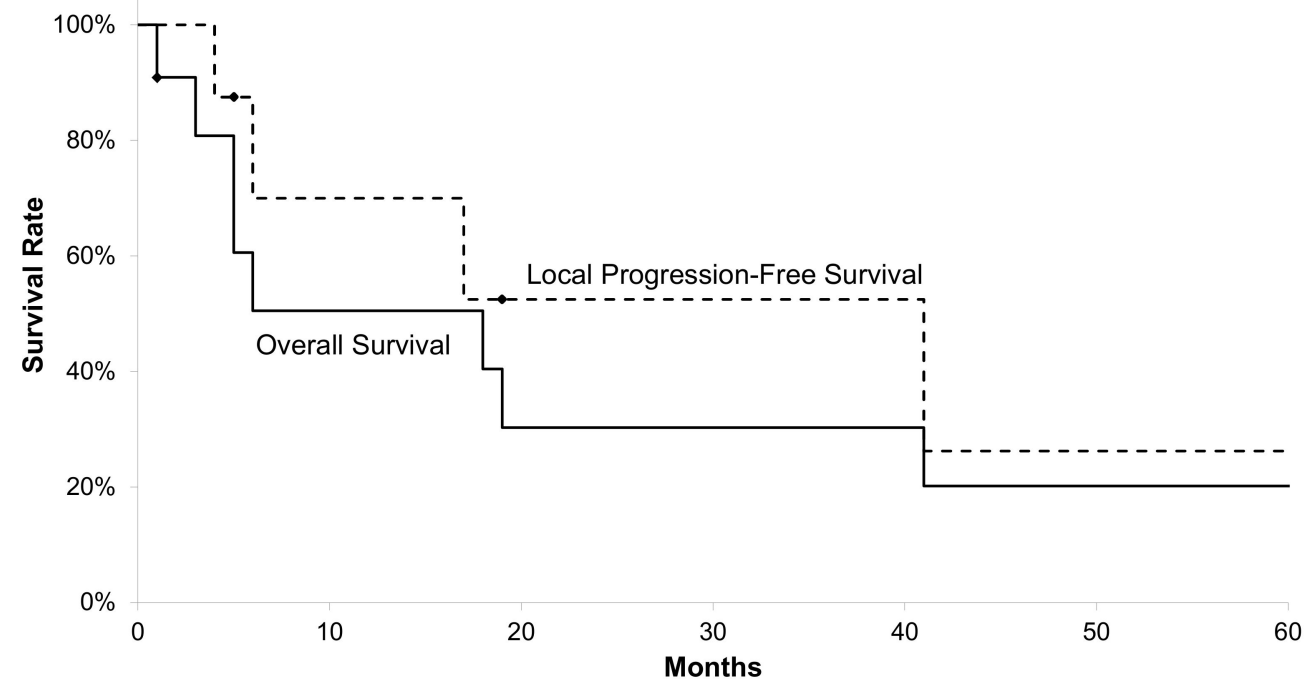

Figure 2. Overall survival and local progression-free survival. Overall survival (OS) in all patients and local progression-free survival (L-PFS) in patients who achieved local complete response or partial response with irradiation are shown. The median OS and L-PFS were 6 and 11.5 months, respectively. One-year OS and L-PFS rates were 51\% (95\% CI, 20-81\%) and 80\% (95\% CI, 34-100\%), respectively. 


\subsection{Toxicities}

Table 2 lists the EBRT-related toxicities. Since this was a retrospective study, we could not assess laboratory data regarding thyroid hormones; therefore, we could not evaluate thyroid hormone complications. Acute Grade 3 complications occurred in five (45\%) patients, including dermatitis $(n=4)$, pharyngeal mucositis $(n=2)$, anemia $(n=1)$, and leukopenia $(n=1)$. Five patients who underwent concurrent treatment using systemic chemotherapy and TKI experienced any grade hematologic complications. In contrast, patients who were irradiated without systemic therapy did not have hematological complications. There were no cases of Grade 4 or 5 acute toxicity and Grade 3 or higher late complications. Two patients who were concurrently or sequentially treated with EBRT and TKI did not develop any severe toxicities.

Table 2. Toxicities related to EBRT therapy.

\begin{tabular}{cccccc}
\hline Toxicities & \multicolumn{5}{c}{ Number of Patients (\%) } \\
\cline { 2 - 5 } & G1 & G2 & G3 & G4 & G5 \\
\hline Hematological & & & & \\
Anemia & $2(18 \%)$ & $2(18 \%)$ & $1(9 \%)$ & $0(0 \%)$ & $0(0 \%)$ \\
Leucopenia & $1(9 \%)$ & $0(0 \%)$ & $1(9 \%)$ & $0(0 \%)$ & $0(0 \%)$ \\
Thrombocytopenia & $0(0 \%)$ & $1(9 \%)$ & $0(0 \%)$ & $0(0 \%)$ & $0(0 \%)$ \\
Non-hematological & & & & & \\
Dermatitis & $4(36 \%)$ & $3(27 \%)$ & $3(27 \%)$ & $0(0 \%)$ & $0(0 \%)$ \\
Pharyngitis & $0(0 \%)$ & $2(18 \%)$ & $2(18 \%)$ & $0(0 \%)$ & $0(0 \%)$ \\
Nausea & $1(9 \%)$ & $0(0 \%)$ & $0(0 \%)$ & $0(0 \%)$ & $0(0 \%)$ \\
\hline
\end{tabular}

G: grade, according to the CTCAE version 5.0.

\section{Discussion}

This retrospective study investigated the treatment outcomes of EBRT for LATC and found that EBRT with or without systemic therapy could achieve local CR+PR in $73 \%$ of the included patients with LATC, among whom $75 \%$ achieved a $\geq 30 \%$ tumor size reduction within 6 months. Grade 3 complications occurred in $5(45 \%)$ patients, with none of the patients having Grade 4 or 5 complications. Our results suggest that EBRT is an effective and safe treatment for LATC.

In this study, $75 \%$ of patients achieved $\geq 30 \%$ tumor size reduction without presenting severe toxicities. This retrospective study lacked data regarding post-EBRT thyroid hormone levels; therefore, we could not investigate toxicities related to thyroid hormones. Nonetheless, thyroid gland irradiation has been reported to decrease thyroid hormone levels [16,17]. Therefore, there is a need for careful examination for late thyroid toxicity and dysfunction. Regarding the treatment approach for thyroid cancer, it is crucial to consider both survival benefits and local tumor reduction. Specifically, the latter endpoint is important given the risk of mass effect and compression or invasion to the trachea or major vessels, which could precipitate a medical emergency. We found that tumor size reduction could be achieved in $75 \%$ of patients treated with EBRT within 6 months of treatment completion. A secondary analysis of a phase 3 trial on lenvatinib for radioiodine-refractory differentiated thyroid cancer (SELECT trial) reported that the median maximum percentage change in tumor size was $-42.9 \%$, with a median tumor reduction of $-24.7 \%$ at 8 postrandomization weeks [18]. Therefore, lenvatinib yields a strong tumor response. However, irradiation may be the preferred treatment option for tumor reduction in patients where TKI is deemed unsuitable, such as those who present a high risk of severe complications or a fragile condition.

In our study, two patients were treated with TKI, concurrently or after EBRT, without presenting severe toxicities. Few studies have investigated the concurrent or sequential use of TKI with EBRT for LATC. In vitro studies have reported that combination therapy with irradiation and lenvatinib significantly inhibited thyroid cancer growth by inducing apoptosis and G2/M phase cell cycle arrest and increasing cellular uptake of lenvatinib into 
cancer cells [19]. Some clinical reports have highlighted the positive treatment effects with the concurrent use of TKI and EBRT for thyroid cancer without severe toxicities [20,21]. However, other reports have shown that TKI could induce severe toxicities in patients with extended tumor invasion to the carotid artery or a history of neck irradiation [7,22]. No prospective trials have investigated the combined therapy with irradiation and lenvatinib for thyroid cancer; therefore, there is a need for careful consideration and patient discussion before clinically applying this combined therapy.

In this study, a conventional dose schedule using 3D-conformal radiotherapy with $X$-ray was employed. With the evolution of irradiation techniques, a higher radiation dose can be administered to target lesions with less exposure to the surrounding normal tissues and organs. Total radiation doses $>45 \mathrm{~Gy}$ have been reported to be associated with good prognosis in anaplastic thyroid carcinoma [11]. Furthermore, a cumulative dose $>64$ Gy may be more effective than lower doses in achieving locoregional control [9]. However, it can be difficult to use such high doses with conventional techniques, especially for bulky tumors surrounding high-risk organs, including the spinal cord and esophagus, which lack tolerability for such high radiation doses. In these situations, intensity-modulated radiation therapy (IMRT) could be considered for delivering high radiation doses while reducing late side effects, including esophageal stricture and subglottic laryngeal stenosis [10,12]. Although there remains no evidence regarding proton beam therapy and carbon ion therapy for unresectable thyroid cancer, from a perspective of radiation physics, they could improve dose distribution for higher radiation doses compared with X-ray therapy, without increasing radiation exposure of the surrounding normal tissues and organs. A phase II trial reported that boron neutron capture therapy (BNCT), which is a cancer cellspecific radiation therapy involving high linear energy transfer alpha rays, was effective for locally advanced or recurrent head and neck cancer [23]. Although this previous trial did not include patients with thyroid cancer, BNCT may allow safe delivery of high-dose radiation for thyroid tumors. In addition to increasing the total dose, there have been studies on increasing the daily dose and shortening the treatment period. Accelerated hyperfractionated radiotherapy, which involves irradiation twice a day with a shorter total treatment period and is effective for small cell lung carcinoma [24], has been applied with the aim of improving treatment outcomes in anaplastic thyroid cancer, although there was no significant survival benefit [25]. Further investigation using these new techniques or ingenuity of radiation schedules is needed to improve the treatment outcomes of EBRT for unresectable thyroid cancer.

This study had two important limitations. First, this was a small-scale, retrospective, single-center study, which could have limited our findings. Given that EBRT for LATC is not well established, the opportunity for delivering EBRT to patients with LATC is rare. Our search for patients who met the inclusion criteria during a 15-year period yielded only 11 cases. To evaluate treatment outcomes of EBRT for LATC, a multicenter prospective study is necessary. The second limitation pertains to the variability in the irradiation dose and field, with missing data regarding tumor size and thyroid hormone function. Uniformed radiation planning with respect to the radiation dose and target should be applied to compare treatment outcomes. Moreover, there could have been unidentified cases of late toxicity of thyroid hormone. Despite these limitations, our results suggest that EBRT for LATC has a tumor-reductive effect without producing severe toxicities. There is a need for further prospective studies that employ advanced techniques, including IMRT, charged-particle therapies, and BNCT, as well as ingenuity of radiation schedules, including accelerated hyperfractionated radiotherapy and hypofractionated therapy, with higher doses per fraction for improving the treatment outcomes of EBRT for unresectable thyroid cancer. 


\title{
5. Conclusions
}

In conclusion, EBRT achieved tumor size reduction in $73 \%$ of patients, among whom $75 \%$ had tumor size reduction within 6 months of EBRT completion. Treatment was well tolerated with few complications. Although TKIs are the main therapy for unresectable thyroid cancer, EBRT can be considered in cases with a clinical risk of using TKIs.

\begin{abstract}
Author Contributions: Conceptualization: Y.W. and A.A.; methodology: Y.W., A.A., and M.H.; software: Y.W.; validation: Y.W., A.A. and S.K.; formal analysis: Y.W.; investigation: Y.W., S.K., E.O. and N.T.; resources: Y.W.; data curation: Y.W., S.K., E.O. and N.T.; writing-original draft preparation, Y.W.; writing-review and editing, Y.W., A.A. and M.H.; visualization, Y.W.; supervision, Y.W., A.A. and M.H.; project administration, Y.W.; funding acquisition, none. All authors have read and agreed to the published version of the manuscript.
\end{abstract}

Funding: This research received no external funding.

Institutional Review Board Statement: The study was conducted according to the guidelines of the Declaration of Helsinki, and approved by the Institutional Review Board (or Ethics Committee) of Akita University Hospital (no. 1951, approved on 27 April 2018).

Informed Consent Statement: Patient consent was waived since this study used anonymized data.

Data Availability Statement: The data presented in this study will be provided upon reasonable request.

Conflicts of Interest: The authors declare no conflict of interest.

\section{References}

1. Filetti, S.; Durante, C.; Hartl, D.; Leboulleux, S.; Locati, L.D.; Newbold, K.; Papotti, M.G.; Berruti, A. Thyroid cancer: ESMO Clinical Practice Guidelines for diagnosis, treatment and follow-up. Ann. Oncol. 2019, 30, 1856-1883. [CrossRef]

2. Haugen, B.R.; Alexander, E.K.; Bible, K.C.; Doherty, G.M.; Mandel, S.J.; Nikiforov, Y.E.; Pacini, F.; Randolph, G.W.; Sawka, A.M.; Schlumberger, M.; et al. 2015 American Thyroid Association Management Guidelines for Adult Patients with Thyroid Nodules and Differentiated Thyroid Cancer: The American Thyroid Association Guidelines Task Force on Thyroid Nodules and Differentiated Thyroid Cancer. Thyroid 2016, 26, 1-133. [CrossRef] [PubMed]

3. Ito, Y.; Onoda, N.; Okamoto, T. The revised clinical practice guidelines on the management of thyroid tumors by the japan associations of endocrine surgeons: Core questions and recommendations for treatments of thyroid cancer. Endocr. J. 2020, 67, 669-717. [CrossRef] [PubMed]

4. Schlumberger, M.; Tahara, M.; Wirth, L.J.; Robinson, B.; Brose, M.S.; Elisei, R.; Habra, M.A.; Newbold, K.; Shah, M.H.; Hoff, A.O.; et al. Lenvatinib versus placebo in radioiodine-refractory thyroid cancer. N. Engl. J. Med. 2015, 372, 621-630. [CrossRef]

5. Brose, M.S.; Nutting, C.M.; Jarzab, B.; Elisei, R.; Siena, S.; Bastholt, L.; De La Fouchardiere, C.; Pacini, F.; Paschke, R.; Shong, Y.K.; et al. Sorafenib in radioactive iodine-refractory, locally advanced or metastatic diff erentiated thyroid cancer: A randomised, double-blind, phase 3 trial. Lancet 2014, 384, 319-328. [CrossRef]

6. Wells, S.A.; Robinson, B.G.; Gagel, R.F.; Dralle, H.; Fagin, J.A.; Santoro, M.; Baudin, E.; Elisei, R.; Jarzab, B.; Vasselli, J.R.; et al. Vandetanib in patients with locally advanced or metastatic medullary thyroid cancer: A randomized, double-blind phase III trial. J. Clin. Oncol. 2012, 30, 134-141. [CrossRef] [PubMed]

7. Obata, K.; Sugitani, I.; Ebina, A.; Sugiura, Y.; Toda, K.; Takahashi, S.; Kawabata, K. Common carotid artery rupture during treatment with lenvatinib for anaplastic thyroid cancer. Int. Cancer Conf. J. 2016, 5, 197-201. [CrossRef]

8. Staub, Y.; Nishiyama, A.; Suga, Y.; Fujita, M.; Matsushita, R.; Yano, S. Clinical characteristics associated with lenvatinib-induced fistula and tumor-related bleeding in patients with thyroid cancer. Anticancer Res. 2019, 39, 3871-3878. [CrossRef]

9. Meadows, K.M.; Amdur, R.J.; Morris, C.G.; Villaret, D.B.; Mazzaferri, E.L.; Mendenhall, W.M. External beam radiotherapy for differentiated thyroid cancer. Am. J. Otolaryngol. Head Neck Med. Surg. 2006, 27, 24-28. [CrossRef] [PubMed]

10. Schwartz, D.L.; Lobo, M.J.; Ang, K.K.; Morrison, W.H.; Rosenthal, D.I.; Ahamad, A.; Evans, D.B.; Clayman, G.; Sherman, S.I.; Garden, A.S. Postoperative external beam radiotherapy for differentiated thyroid cancer: Outcomes and morbidity with conformal treatment. Int. J. Radiat. Oncol. Biol. Phys. 2009, 74, 1083-1091. [CrossRef]

11. Pierie, J.P.E.N.; Muzikansky, A.; Gaz, R.D.; Faquin, W.C.; Ott, M.J. The effect of surgery and radiotherapy on outcome of anaplastic thyroid carcinoma. Ann. Surg. Oncol. 2002, 9, 57-64. [CrossRef]

12. Rosenbluth, B.D.; Serrano, V.; Happersett, L.; Shaha, A.R.; Tuttle, R.M.; Narayana, A.; Wolden, S.L.; Rosenzweig, K.E.; Chong, L.M.; Lee, N.Y. Intensity-modulated radiation therapy for the treatment of nonanaplastic thyroid cancer. Int. J. Radiat. Oncol. Biol. Phys. 2005, 63, 1419-1426. [CrossRef]

13. Terezakis, S.A.; Lee, K.S.; Ghossein, R.A.; Rivera, M.; Tuttle, R.M.; Wolden, S.L.; Zelefsky, M.J.; Wong, R.J.; Patel, S.G.; Pfister, D.G. Role of external beam radiotherapy in patients with advanced or recurrent nonanaplastic thyroid cancer: Memorial Sloan-Kettering Cancer Center experience. Int. J. Radiat. Oncol. Biol. Phys. 2009, 73, 795-801. [CrossRef] 
14. Brierley, J.D.; Gospodarowicz, M.K.; Wittekind, C. TNM Classification of Malignant Tumours, 8th ed.; Blackwell Publishing: Hoboken, NJ, USA, 2017.

15. Edward, C.H. Liner-Quadratic Equation. In Perez E Brady's Principles and Practice of Radiation Oncology, 7th ed.; Wolters Kluwer: Alphen aan den Rijn, The Netherlands, 2018; pp. 27-30.

16. Bertok, L.; Nagy, S.U. Effect of irradiation on serum T4 level and response of thyroid gland to exogenous TSH in rats. J. Radiat. Res. 1986, 27, 225-229. [CrossRef] [PubMed]

17. Nagayama, Y. Radiation-related thyroid autoimmunity and dysfunction. J. Radiat. Res. 2018, 59, ii98-ii107. [CrossRef] [PubMed]

18. Robinson, B.; Schlumberger, M.; Wirth, L.J.; Dutcus, C.E.; Song, J.; Taylor, M.H.; Kim, S.B.; Krzyzanowska, M.K.; Capdevila, J.; Sherman, S.I. Characterization of tumor size changes over time from the phase 3 study of lenvatinib in thyroid cancer. J. Clin. Endocrinol. Metab. 2016, 101, 4103-4139. [CrossRef] [PubMed]

19. Suzuki, K.; Iwai, H.; Utsunomiya, K.; Kono, Y.; Kobayashi, Y.; Van Bui, D.; Sawada, S.; Yun, Y.; Mitani, A.; Kondo, N.; et al. Combination therapy with lenvatinib and radiation significantly inhibits thyroid cancer growth by uptake of tyrosine kinase inhibitor. Exp. Cell Res. 2021, 398, 112390. [CrossRef]

20. Wada, Y. A case of unresectable squamous cell carcinoma of thyroid gland which could be local controlled by lenvatinib combined with radiotherapy. Jpn. J. Clin. Radiol. 2016, 61, 953-958.

21. Yamazaki, T. Combined use of lenvatinib and external irradiation for undifferentiated thyroid cancer in aged patients with multiple comorbidities: A case study. J. Jpn. Thyroid Assoc. 2018, 9, 69-73. [CrossRef]

22. Shimo, T.; Yoshidome, K.; Yasuno, K.; Tori, M. Three cases of recurrent thyroid cancer involving the perforation of adjacent structures caused by lenvatinib after external radiation therapy. J. Jpn. Surg. Assoc. 2018, 79, 283-288. [CrossRef]

23. Hirose, K.; Konno, A.; Hiratsuka, J.; Yoshimoto, S.; Kato, T.; Ono, K.; Otsuki, N.; Hatazawa, J.; Tanaka, H.; Takayama, K.; et al. Boron neutron capture therapy using cyclotron-based epithermal neutron source and borofalan (10B) for recurrent or locally advanced head and neck cancer (JHN002): An open-label phase II trial. Radiother. Oncol. 2021, 155, 182-187. [CrossRef] [PubMed]

24. Turrisi, A.T., 3rd; Kim, K.; Blum, R.; Sause, W.T.; Livingston, R.B.; Komaki, R.; Wagner, H.; Aisner, S.; Johnson, D.H. Twicedaily compared with once-daily thoracic radiotherapy in limited small-cell lung cancer treated concurrently with cisplatin and etoposide. N. Engl. J. Med. 1999, 340, 265-271. [CrossRef] [PubMed]

25. Dandekar, P.; Harmer, C.; Barbachano, Y.; Rhys-Evans, P.; Harrington, K.; Nutting, C.; Newbold, K. Hyperfractionated Accelerated Radiotherapy (HART) for anaplastic thyroid carcinoma: Toxicity and survival analysis. Int. J. Radiat. Oncol. Biol. Phys. 2009, 74, 518-521. [CrossRef] [PubMed] 